\title{
Bone marrow-derived mesenchymal stem cells attenuate acute liver injury and regulate the expression of fibrinogen-like-protein 1 and signal transducer and activator of transcription 3
}

\author{
ZHUOLIN ZOU ${ }^{1}$, YIJING CAI $^{1}$, YI CHEN $^{1}$, SI CHEN $^{1}$, LIYUAN LIU $^{1}$, \\ ZHONGHAI SHEN ${ }^{2}$, SAINAN ZHANG ${ }^{1}$, LANMAN XU ${ }^{1}$ and YONGPING CHEN ${ }^{1}$
}

\begin{abstract}
${ }^{1}$ Department of Infectious Disease, Wenzhou Key Laboratory of Hepatology, Hepatology Institute of Wenzhou Medical University, The First Affiliated Hospital of Wenzhou Medical University, Wenzhou, Zhejiang 325000; ${ }^{2}$ Department of Orthopedic Surgery, The Second Affiliated Hospital of Wenzhou Medical University, Wenzhou, Zhejiang 325027, P.R. China
\end{abstract}

Received June 9, 2014; Accepted February 27, 2015

DOI: $10.3892 / \mathrm{mmr} .2015 .3660$

\begin{abstract}
In recent years, bone marrow-derived mesenchymal stem cells (BMSCs) have been demonstrated to exert extensive therapeutic effects on acute liver injury; however, the underlying mechanisms of these effects have remained to be elucidated. The present study focused on the potential anti-apoptotic and pro-regenerative effects of BMSCs in D-galactosamine (D-Gal) and lipopolysaccharide (LPS)-induced acute liver injury in rats. An experimental rat acute liver injury model was established by intraperitoneal injection of D-Gal $(400 \mathrm{mg} / \mathrm{kg})$ and LPS $(80 \mu \mathrm{g} / \mathrm{kg})$. BMSCs and an identical volume of saline were administered via the caudal vein $2 \mathrm{~h}$ after the D-Gal and LPS challenge. Subsequently, the serum samples were collected to detect the levels of alanine aminotransferase and aspartate aminotransferase. Hematoxylin and eosin staining, terminal deoxynucleotidyl transferase-mediated nick-end labeling assay and immunohistochemical staining were performed to determine apoptosis, regeneration and histological changes of liver sections. Western blotting and reverse transcription-quantitative polymerase chain reaction were performed to detect the protein and mRNA expression levels of fibrinogen-like-protein 1 (FGL1), phosphorylated signal transducer and activator of transcription 3 (p-STAT3), STAT3 and B-cell lymphoma $2(\mathrm{Bcl}-2)$ and Bcl-2 associated $\mathrm{X}$ protein $(\mathrm{Bax})$ in liver tissue samples. The results indicated that intravenous transplantation of BMSCs significantly
\end{abstract}

Correspondence to: Dr Yongping Chen, Department of Infectious Disease, Wenzhou Key Laboratory of Hepatology, Hepatology Institute of Wenzhou Medical University, The First Affiliated Hospital of Wenzhou Medical University, 2 Fuxue Road, Wenzhou, Zhejiang 325000, P.R. China

E-mail: 13505777281@163.com

Key words: acute liver injury, bone marrow-derived mesenchymal stem cells, fibrinogen-like-protein 1, signal transducer and activator of transcription 3 decreased the levels of alanine aminotransferase and aspartate aminotransferase, and reduced hepatocellular necrosis and inflammatory cell infiltration. Additionally, a terminal deoxynucleotidyl transferase-mediated nick-end labeling assay and immunohistochemical staining revealed that BMSC treatment reduced hepatocyte apoptosis and enhanced liver regeneration. Furthermore, Bcl-2 expression was increased, whilst the protein expression of Bax was reduced. The expression of FGL1 and p-STAT3 were elevated concurrently with the improvement of liver function. These results demonstrated that BMSCs may provide a promising potential agent for the prevention of acute liver injury via inhibition of hepatocyte apoptosis and acceleration of liver regeneration. The mechanism may be, a least in part, a consequence of the upregulation of FGL1 expression and the induction of STAT3 phosphorylation.

\section{Introduction}

The liver is vulnerable to tissue injury due to its anatomical position as well as its function, which involve exposure to various aberrant factors containing hepatotropic viruses, ethanol (1) and medicines (2), and may result in liver injury. Acute liver injury is characterized by liver dysfunction and may progress into liver failure in the absence of intervention at an early stage (3). Once this dysfunction is aggravated and exacerbated into liver failure, it becomes a disease associated with high rates of mortality, often requiring liver transplantation (4). However, liver transplantation is limited due to the critical shortage of liver donors, high economic costs and risk of transplant rejection (5). In recent years, cellular therapy has been demonstrated to be a prospective treatment for acute liver injury, in animal experiments and cellular level investigations, through resisting hepatocyte apoptosis and promoting liver tissue regeneration $(6,7)$.

Bone-marrow-derived mesenchymal stem cells (BMSCs) have been reported to be able to treat numerous types of disease in animals, including acute kidney failure (8), ischemic heart disease (9), lung injury (10) and intracerebral hemorrhage (11), based on their biological characteristics. Multiple studies have demonstrated that BMSCs are able to migrate to impaired 
tissues in response to damage, including necrosis (12), hypoxia (13) and ischemia (14). The mechanisms by which BMSCs alleviate acute liver injury involve challenges. It has primarily been reported that BMSCs are able to differentiate into hepatocyte-like cells and thereby promote liver tissue recovery and healing (15). However, there is increasing awareness of the contribution of BMSC-induced generation of trophic molecules by paracrine action in the alleviation of acute liver injury. BMSCs migrate to the injured tissue site and secrete a broad range of cytokines, growth factors and chemokines, which exert anti-necrotic, anti-apoptotic and anti-inflammatory effects under injury conditions (16-18). However, the specific molecular mechanisms underlying the involvement of BMSCs in acute liver injury have not been sufficiently explored.

Recently, Xagorari et al (19) demonstrated that mesenchymal stem cell-conditioned medium enhanced the expression of fibrinogen-like-protein 1 (FGL1) in cultured mouse hepatoycytes, following the induction of acute liver injury. FGL1 (also termed FREP1 or hepassocin) is a protein secreted by hepatocytes, which is a member of the fibrinogen family (20). Studies have shown that FGL1 is implicated in liver injury as a mitogenic growth factor and may facilitate damaged liver tissue regeneration and downregulate the expression of proapoptotic factors (21-23). In the present study, it was hypothesized that intravenous transplantation of BMSCs may increase the expression of FGL1 in the injured rat liver and rescue the liver following acute injury. By determination of dynamic changes in B cell lymphoma 2 (Bcl-2) and $\mathrm{Bcl}-2$ associated $\mathrm{X}$ protein (Bax) expression, as well as hepatocyte apoptosis, the effect of BMSCs on apoptosis following acute rat liver injury was investigated. The expression of proliferating cell nuclear antigen (PCNA) in liver tissues was also evaluated in order to determine the role of BMSCs in liver regeneration. To further investigate the mechanism of BMSCs in the treatment of acute liver injury, FGL1, phosphorylated signal transducer and activator of transcription 3 (p-STAT3) and STAT3 protein expression levels were detected. The results of the present study may lay the foundation for further exploration of the mechanisms underlying BMSC transplantation in the treatment of acute liver injury.

\section{Materials and methods}

Animals. Seventy-six male specific pathogen-free (SPF) Sprague-Dawley (SD) rats (8 weeks old; 250-300 g) were acquired from the Shanghai Laboratory Animal Center (Shanghai, China). All rats were fed under SPF conditions and had free access to food and water in a standardized laboratory. The rats were housed at $20-26^{\circ} \mathrm{C}$ and $40-70 \%$ relative humidity, and underwent a $12 \mathrm{~h} / 12 \mathrm{~h}$ light/dark cycle. Experiments and procedures were approved by the Institutional Animal Committee of Wenzhou Medical University (Wenzhou, China).

Isolation and culture of BMSCs. The isolation of BMSCs was conducting using the adherence method $(24,25)$. Four healthy male SPF SD rats ( 8 weeks old) were sacrificed by intraperitoneal injection of $10 \%$ chloral hydrate $(3 \mathrm{ml} / \mathrm{kg}$; Shanghai Chemical Reagent Co., Ltd., Shanghai, China) and placed in $70 \%$ alcohol for sterilization prior to the dissection of the bilateral femur and tibia. The adjunctive muscle and soft tissue were stripped under axenic conditions and the marrow cavity was flushed slowly with a $10-\mathrm{ml}$ injection syringe filled with $\alpha$-minimum essential medium ( $\alpha$-MEM; Thermo Fisher Scientific, Waltham, MA, USA). The mixture was filtered through a 200-mesh screen filter (Shanghai Rego Biotechnology Co., Ltd, Shanghai, China) and the filtered solution was centrifuged at $206 \mathrm{x}$ g for $5 \mathrm{~min}$ (Thermo Fisher Scientific). The supernatant was discarded and cells were resuspended in liquid culture medium with $\alpha$-MEM containing $10 \%$ fetal bovine serum (Gibco Life Technologies, Grand Island, NY, USA). Cells were seeded in 50- $\mathrm{cm}^{2}$ flasks (Corning Inc., Corning, NY, USA) at $37^{\circ} \mathrm{C}$ in a $5 \% \mathrm{CO}_{2}$ humidified atmosphere. Following $48 \mathrm{~h}$ of incubation, cells which were not adherent were washed away with sterile saline solution. Subsequently, the medium was changed every 3-4 days. The cultured cells were digested with $0.25 \%$ trypsin (Gibco Life Technologies) with a passage ratio of 1:2 and the third generation of BMSCs was sent to the Institute of Medical Sciences of The First Affiliated Hospital of Wenzhou Medical University (Wenzhou, China) for flow cytometric analysis (FACSCalibur; BD Biosciences, Franklin Lakes, NJ, USA). The forth generation of BMSCs were digested by $0.25 \%$ trypsin (Gibco Life Technologies) and used to generate a cell suspension in $9 \%$ sodium chloride solution (Shanghai Chemical Reagent Co., Ltd.) with a density of $1.0 \times 10^{7}$ cells $/ \mathrm{ml}$ for experimental use.

Flow cytometric analysis. The third generation of BMSCs were harvested and incubated with the following phycoerythrin-conjugated antibodies: CD45 (cat. no. 202207), CD29 (cat. no. 102207), CD11b (cat. no. 101207), and CD90 (cat. no. 205903 ) (BioLegend, Inc., San Diego, CA, USA) at room temperature for $15 \mathrm{~min}$. The cells were subsequently washed twice with PBS (Gibco Life Technologies) and were sent to the Institute of Medical Sciences of The First Affiliated Hospital of Wenzhou Medical University (Wenzhou, China) for flow cytometric analysis (FACSCalibur; BD Biosciences, Franklin Lakes, NJ, USA).

Groups and experimental design. The remaining 72 SD rats were randomly divided into two groups (the control group and the BMSCs treatment group; $n=36$ per group, 12 per time point). The rats of the two experimental groups were injected with lipopolysaccharide (LPS; $80 \mathrm{ug} / \mathrm{kg}$; Sigma-Aldrich, St. Louis, MO, USA) and D-galactosamine (D-Gal; $400 \mathrm{mg} / \mathrm{kg}$; Sigma-Aldrich), and two hours following this injection, rats in the BMSC treatment group were injected with $0.5 \mathrm{ml}\left(5.0 \times 10^{6}\right.$ cells $)$ BMSC suspension via the tail vein and the control group were injected with an identical volume of saline. At 24, 72 and $120 \mathrm{~h}$ following the injection of BMSCs or saline, rats were sacrificed by intraperitoneal injection of $10 \%$ chloral hydrate $(3 \mathrm{ml} / \mathrm{kg})$, blood samples and liver tissues were prepared for further experiments.

Detection of alanine aminotransferase (ALT) and aspartate aminotransferase (AST). In order to determine the degree of liver injury, the levels of serum transaminase were measured. Rats were anesthetized by intraperitoneal injection of $10 \%$ chloral hydrate $(1.5 \mathrm{ml} / \mathrm{kg})$, and $2 \mathrm{ml}$ blood was collected 
via the inferior vena cava. Following standing for $3 \mathrm{~min}$, the samples were centrifuged at 2,400 x $\mathrm{g}$ for $10 \mathrm{~min}$ and the upper serum was kept and stored in a $-80^{\circ} \mathrm{C}$ deep-freezer (Thermo Fisher Scientific). The levels of AST and ALT in the serum were measured with an automatic biochemistry analyzer (KT6HB560; Abbott Laboratories, Chicago, IL, USA).

Histological analysis. Liver tissues were fixed in $4 \%$ neutral formaldehyde solution (Shanghai Sangon Biotechnology Co., Ltd., Shanghai, China) embedded in paraffin (Shanghai Sangon Biotechnology Co., Ltd.), cut into 5- $\mu \mathrm{m}$ sections and viewed under an optical microscope (Nikon Eclipse; Nikon Corp., Tokyo, Japan) to observe pathological changes following staining with hematoxylin and eosin (Shanghai Chemical Reagent Co., Ltd).

Detection of apoptotic hepatocytes in liver tissues. A terminal deoxynucleotidyl transferase-mediated dUTP nick end labeling (TUNEL) assay (Roche Diagnostics, Basel, Switzerland) was used to detect the apoptosis of hepatocytes in stained tissue slices. According to the operating instructions, tissue sections embedded with paraffin were routinely dewaxed, rehydrated and pretreated with proteinase-K. Following immersion in methanol, the endogenous peroxidase activity was blocked. Subsequently the TUNEL reaction mixture and converter-peroxidase were added. Following staining of the tissue slices with 3,3'-diaminobenzidine (DAB), the number of apoptotic cells was calculated in five randomly selected areas under x400 magnification. Photographs were captured using a digital image-capture system (Nikon Eclipse LV100D).

Immunohistochemical analysis of PCNA. Paraffin-embedded slices were dewaxed, rehydrated and treated with $3 \%$ hydrogen peroxide (Shanghai Sangon Biotechnology Co., Ltd.). Following $10 \mathrm{~min}$ of incubation, the slices were blocked with $5 \%$ bovine serum albumin (BSA; Sigma-Aldrich) in Tris-buffered saline Tween (TBST; Shanghai Sangon Biotechnology Co., Ltd.), and incubated with the primary rabbit monoclonal antibody against PCNA (1:16,000 dilution; Cell Signaling Technology, Inc., Danvers, MA, USA) at $4^{\circ} \mathrm{C}$ overnight. Subsequently, the samples were incubated with peroxidase-conjugated secondary monoclonal antibody (PV-9001; dilution 1:10,000; Zhongshan Goldenbridge Biotechnology Co., Ltd., Beijing, China) at $37^{\circ} \mathrm{C}$ for $20 \mathrm{~min}$. DAB then served as the chromogen and hematoxylin as the counterstain. PBS was used as the negative control, instead of the primary antibody. Sections were examined under a microscope, and nuclei exhibiting a brown color (regardless of staining intensity) were considered to be positive. The number of apoptotic cells was calculated in five randomly selected areas under a microscope (Nikon Eclipse LV100D; magnification, $\mathrm{x} 400$ ).

Protein isolation and western blot analysis. Total protein was isolated using lysis buffer $(1 \mathrm{ml}$ radioimmunoprecipitation pyrolysis liquid, $10 \mu \mathrm{l}$ phenylmethanesulfonylfluoride and $10 \mu \mathrm{l}$ sodium orthovanadate activating agent; Roche Diagnostics) accompanied by a protease inhibitor cocktail obtained from Roche Diagnostics. Samples were placed in an ice bath for $30 \mathrm{~min}$ and centrifuged at 16,000 x $\mathrm{g}$ for
10 min, the supernatant was subsequently collected and stored at $-80^{\circ} \mathrm{C}$ for preservation, and the protein concentration was measured using a bicinchoninic acid protein assay kit (ProteinTech, Wuhan, China). The samples (8 $\mu \mathrm{g}$ protein each) were subjected to $10 \%$ SDS-PAGE following heat denaturation at $95^{\circ} \mathrm{C}$ for $5 \mathrm{~min}$. Subsequently, the target proteins in the gel were transferred onto polyvinylidene difluoride (PVDF) membranes (Bio-Rad Laboratories, Inc., Hercules, CA, USA) and the membranes were blocked with 5\% BSA for $1.5 \mathrm{~h}$ at room temperature. The membranes were incubated with the following primary antibodies: Rabbit polyclonal immunoglobulin G (IgG) anti-FGL1 (sc-55957-R; dilution 1:1,000), mouse monoclonal anti-Bax (sc-7480; dilution 1:200), mouse monoclonal anti-Bcl-2 (sc-7382; dilution 1:200), mouse monoclonal anti-p-STAT3 (sc-8059; dilution 1:1,000), rabbit polyclonal anti-STAT3 (sc-482; dilution 1:1,000) and mouse monoclonal anti-GAPDH (sc-365062; dilution 1:5,000) (Santa Cruz Biotechnology, Inc., Dallas, TX, USA) at $4^{\circ} \mathrm{C}$ overnight. Subsequently, the membranes were washed four times with TBST for $7 \mathrm{~min}$. Following incubation with the HRP-conjugated goat anti-rabbit and anti-rat IgG secondary antibodies (Santa Cruz Biotechnology, Inc.) at room temperature for $1.5 \mathrm{~h}$, the membranes were washed as mentioned above and visualization was performed. Briefly, enhanced chemiluminescence substrate (Pierce Biotechnology, Inc., Rockford, IL, USA) was added to the PVDF membranes. The images were then captured on X-ray film (Eastman Kodak, Rochester, NY, USA), and protein quantification was determined using Gel Pro Analyzer 4 image analysis software (Media Cybernetics, Inc., Rockville, MD, USA).

Reverse transcription-quantitative polymerase chain reaction (qPCR) analysis of FGL1 mRNA expression in liver tissues. Total RNA was extracted using RNAiso Plus reagent (Aidlab Biotechnologies Co., Beijing, China) according to the manufacturer's instructions. To measure the concentration and purity of the RNA, optical density at 260/280 nm was assessed. The primer design was based on the National Institutes of Health Biotechnology Information Center Primer BLAST set (www.ncbi.nlm.nih.gov/tools/primer-blast/) and was conducted by Beijing Keno Leip Biological Technology (Beijing, China). In order to synthesize cDNA, the Power RT kit (Bioteke Corp., Beijing, China) was used, and RT-PCR was conducted on the ABI 7500 Real-Time PCR System (Applied Biosystems Life Technologies, Carlsbad, CA, USA) with SYBR Green fluorescent dye (Toyobo Co., Ltd., Osaka, Japan). The reaction conditions for qPCR were as follows: $95^{\circ} \mathrm{C}$ for $2 \mathrm{~min}$, followed by 40 cycles at $95^{\circ} \mathrm{C}$ for $20 \mathrm{sec}$, $60^{\circ} \mathrm{C}$ for $30 \mathrm{sec}$ and $72^{\circ} \mathrm{C}$ for $30 \mathrm{sec}$, and a final extension step at $72^{\circ} \mathrm{C}$ for $10 \mathrm{~min}$. The relative mRNA abundance was determined using the standard curve method $(26,27)$. The data were analyzed using the $2^{-\Delta \Delta \mathrm{CT}}$ method $(26,27)$. The primers used for RT-qPCR were as follows: FGL1 (144 bp) forward, 5'-GGTGGGCTAGTCACCAAACA-3' and reverse, 5'-CCTTGGTAGTACACGCCGTT-3'; $\beta$-actin (207 bp) forward, 5'-CACCCGCGAGTACAACCTTC-3' and reverse, 5'-CCCATACCCACCATCACACC-3'.

Statistical analysis. SPSS 19.0 statistical software (IBM SPSS, Armonk, NY, USA) was used for statistical analyses 

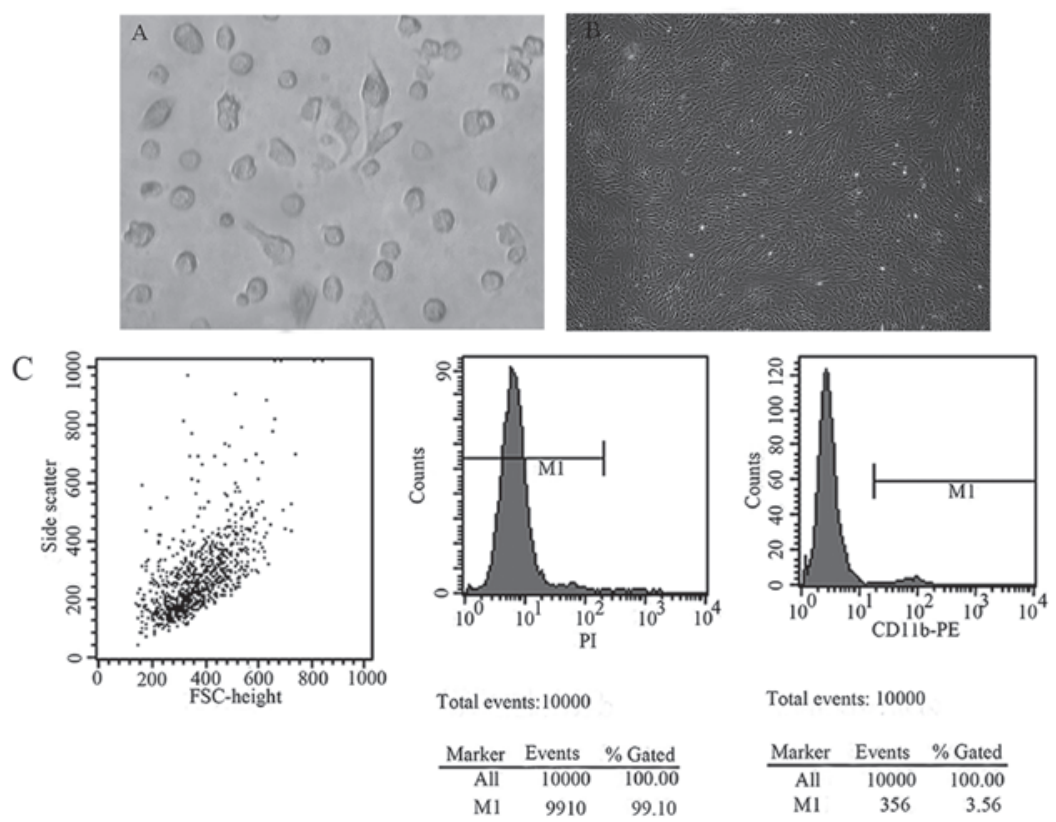

Total events: 10000
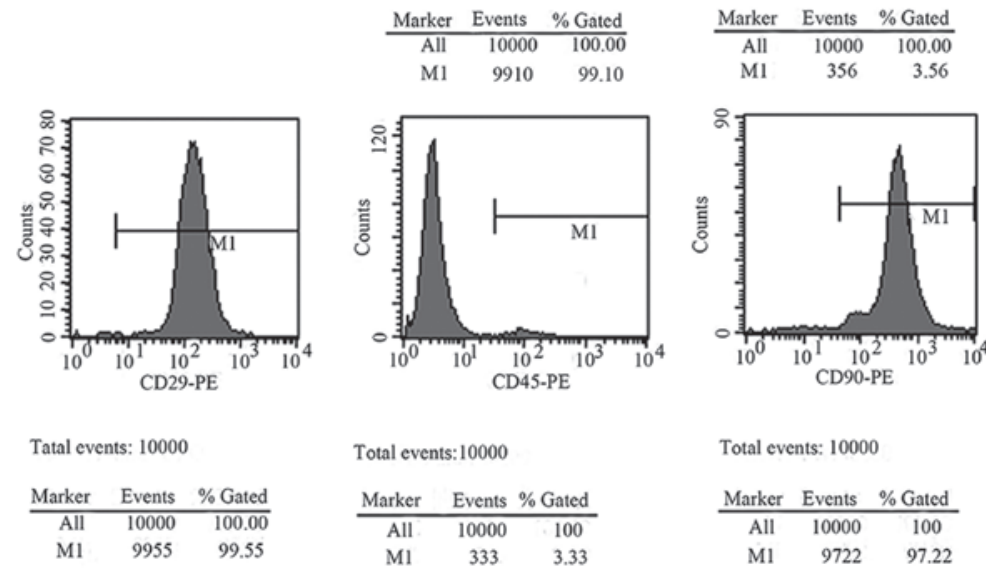

Total events: 10000

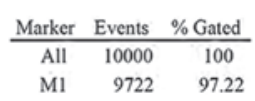

Figure 1. Characterization of BMSCs. (A) BMSCs following three days of culture (F0; magnification, $\mathrm{x} 400)$. (B) BMSCs in the third generation (F3; magnification, x100). (C) Characterization of rat BMSCs by flow cytometric analysis. BMSCs, bone marrow-derived mesenchymal stem cells; PE phycoerythrin.
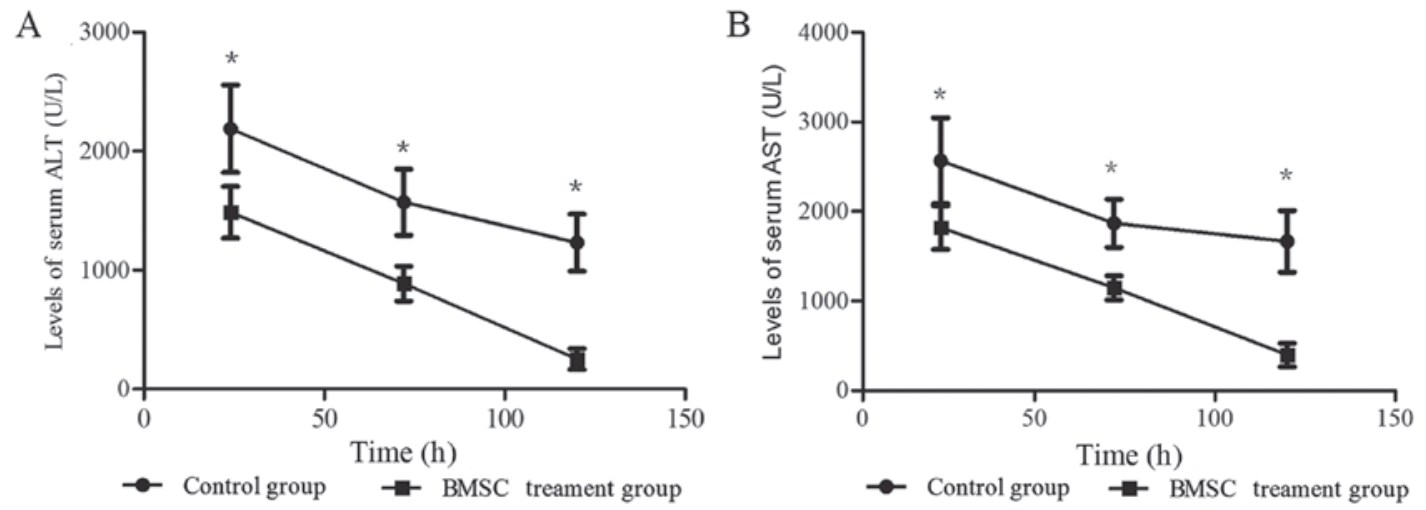

Figure 2. Levels of serum ALT and AST in the control and BMSC treatment groups. (A) ALT levels in peripheral blood samples. (B) AST levels in peripheral blood samples. ${ }^{\mathrm{P}}<0.05$. Values are presented as the mean \pm standard deviation. BMSC, bone marrow-derived mesenchymal stem cell; ALT, alanine aminotransferase; AST, aspartate aminotransferase.

and values are expressed as the mean \pm standard deviation. One-way analysis of variance was employed for statistical comparisons amongst all groups. $\mathrm{P}<0.05$ was considered to indicate a statistically significant difference. The comparative $\mathrm{C}(\mathrm{T})$ method $(26,27)$ was used to estimate the gene expression fold-change.

\section{Results}

Characterization and identification of BMSCs. The extraction of BMSCs was conducted using the adherence method. Following cultivation of primary BMSCs for three days, a small number of cells had adhered to the culture flasks, which 

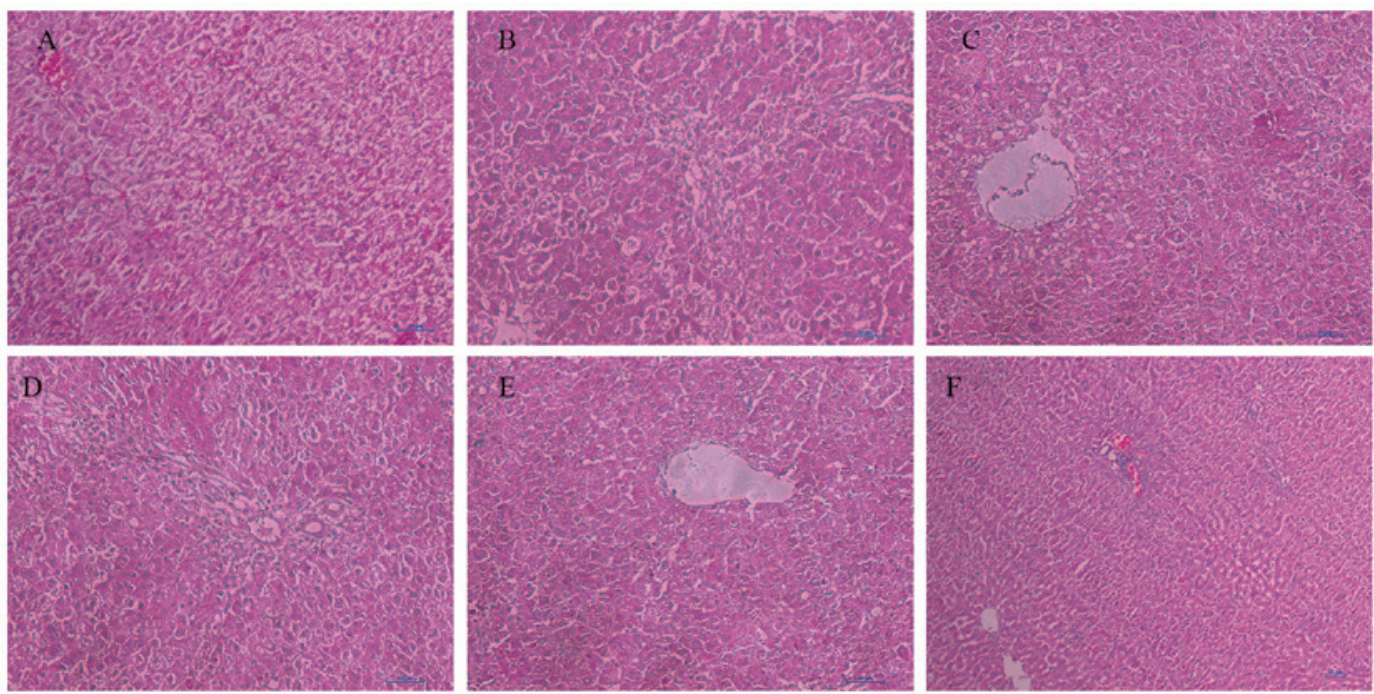

Figure 3. Histological evaluation of liver sections by hematoxylin and eosin staining. (A-C) Control group following 24,72 and $120 \mathrm{~h}$ of incubation, respectively. (D-F) Bone marrow-derived mesenchymal stem cell treatment group following 24, 72 and $120 \mathrm{~h}$ of incubation, respectively. Magnification, x200.
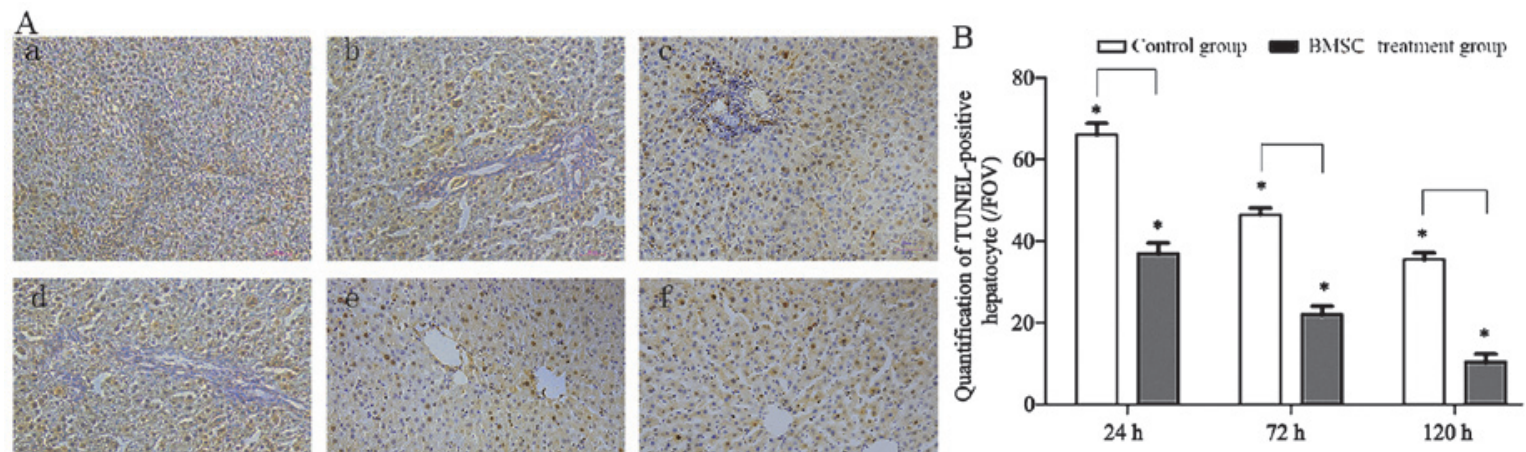

Figure 4. Analysis of apoptosis by TUNEL assay. (A) TUNEL assay of the liver tissues: (a-c) Tissues of the control group 24,72 and $120 \mathrm{~h}$ following treatment, respectively; (d-f) tissues of the BMSC treatment group 24, 72 and $120 \mathrm{~h}$ following treatment, respectively. Magnification, x200. (B) Quantification of TUNEL activated cells. Values are presented as the mean \pm standard deviation; ${ }^{*}<<0.05$. TUNEL, terminal deoxynucleotidyl transferase-mediated dUTP nick-end labeling; BMSCs, bone marrow-derived mesenchymal stem cells; FOV, field of view.

mainly exhibited a short spindle facade (Fig. 1A). Following seven days of culture, cells continuously extended and fused into a single-layered appearance, and most cells displayed a long fusiform or polygon appearance when observed under a microscope. Upon extension to the third generation (Fig. 1B), the morphology of the cells and the light-admitting quality of the cytoplasm were altered and the cells appeared similar to fibroblasts, exhibiting a spiral exterior. The third generation of BMSCs was evaluated by flow cytometry, the results of which indicated that the cells highly expressed CD90 and CD29, whereas the expression levels of CD11b and CD45 were low, demonstrating that BMSCs with a high purity status had been produced (Fig. 1C).

BMSCs inhibit the release of liver enzymes and reduce liver injury. When the population of dead liver cells overcome the regenerating cells during acute liver injury, the liver function becomes out of balance. The levels of ALT and AST released into the peripheral blood indicate the degree of liver damage. In the two experimental groups, the levels of ALT (Fig. 2A) and AST (Fig. 2B) peaked at 24 h. However, the levels of ALT and AST were significantly decreased in the BMSC treatment group at all time-points (Fig. 2), and the minimum levels of ALT and AST in the BMSC treatment group were markedly decreased by $79 \%(\mathrm{P}<0.05)$ and $76 \%(\mathrm{P}<0.05)$ compared with that of the control group at $120 \mathrm{~h}$, respectively. These results indicated that treatment with BMSCs induced a marked decrease in liver enzyme release and demonstrated that the intravenous transplantation of BMSCs may ameliorate the function of the liver in an acute liver injury rat model.

In order to observe the potential effects of BMSC transplantation, histological changes in transplanted and control liver tissues were evaluated by HE staining. The results indicated that the liver tissues of the control group exhibited pronounced congestion and pericentral necrosis of a large area, characteristic of lobular disorder, which was accompanied by significant inflammatory cell infiltration (Fig. 3A). In addition, it was observed that the liver tissues of the control group presented time-dependent injury recovery (Fig. 3B and C); this may be due to the unique regenerative capacity of the liver, 

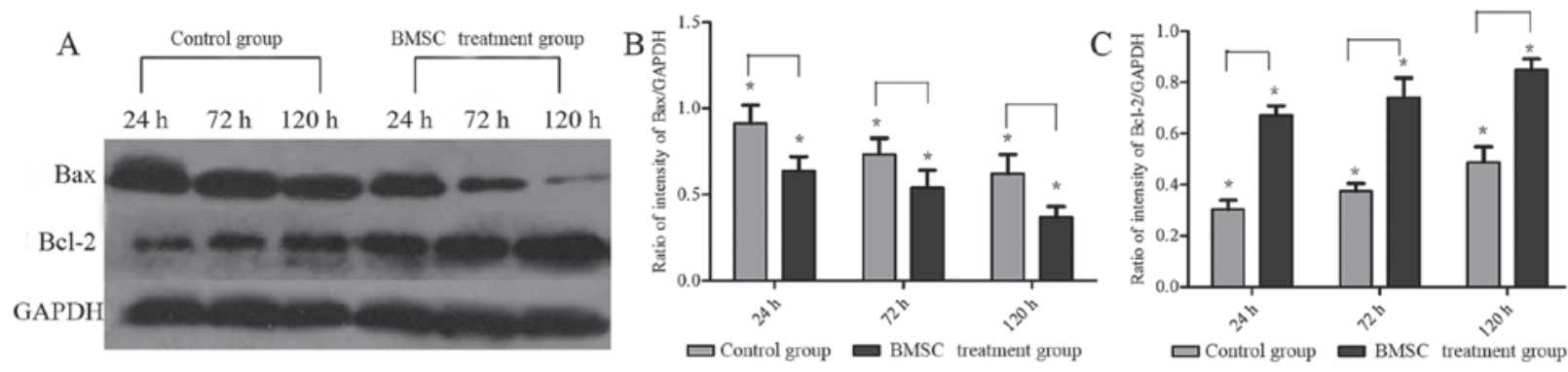

Figure 5. Protein expression of Bax and Bcl-2 in liver tissues. (A) The expression levels of Bax and Bcl-2 proteins were evaluated by western blotting. (B) Quantification of Bax protein expression. (C) Quantification of Bcl-2 protein expression. Values are presented as the mean \pm standard deviation; ${ }^{*} \mathrm{P}<0.05$. GAPDH was used as an internal control. BMSCs, bone marrow-derived mesenchymal stem cells; Bcl-2, B cell lymphoma-2; Bax, Bcl-2-like protein 4; FOV, field of view.
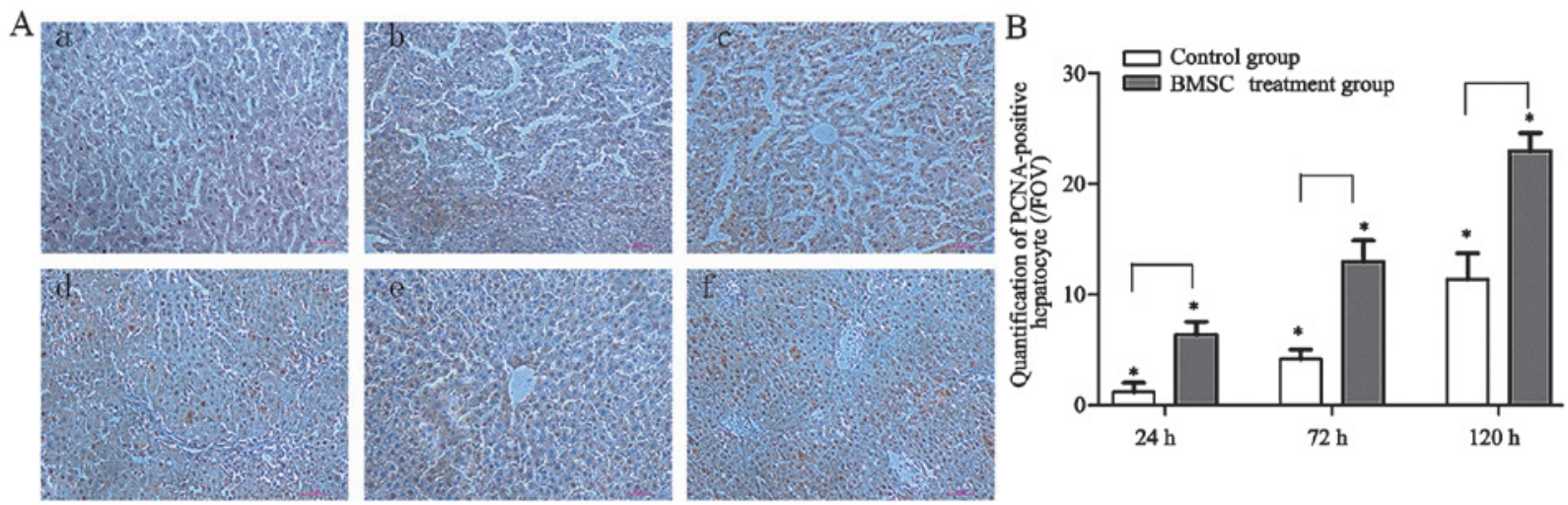

Figure 6. Immohistological staining for PCNA-active nuclei. (A) Immohistological staining of (a-c) control group following 24,72 and $120 \mathrm{~h}$ of treatment, respectively; and (d-f) BMSC treatment group following 24, 72 and $120 \mathrm{~h}$ of treatment, respectively; (magnification, x200). (B) Quantification of PCNA-reactive hepatocytes. Values are presented as the mean \pm standard deviation; ${ }^{*} \mathrm{P}<0.05$. BMSCs, bone marrow-derived mesenchymal stem cells; FOV, field of view; PCNA, proliferating cell nuclear antigen.

meaning that the damaged liver may develop spontaneous recovery. However, these pathomorphological changes were further attenuated by intravenous transplantation of BMSCs, compared with those at the same time-point in the control group. Large tracts of land infiltration of inflammatory cells and necrosis were relieved following the BMSC treatment (Fig. 3D-F).

BMSCs inhibit hepatocellular apoptosis and regulate the expression of apoptosis-associated proteins. In order to verify whether treatment with BMSCs was able to reduce cell apoptosis, a TUNEL assay was performed. The hepatocyte nuclei tissue sections which reacted to TUNEL were calculated according to the aforementioned method. In the control group, numerous sections exhibiting large, apoptotic hepatocyte nuclei were detected (Fig. 4Aa-c); whereas, few were observed in the BMSC treatment group, and this number also significantly decreased with time (Fig. 4Ad-f). In addition, comparisons of the number of TUNEL-reactive hepatocytes between the two groups at the same time-point (Fig. 4B), revealed that the differences were significant at $24(\mathrm{P}<0.05), 72(\mathrm{P}<0.05)$ and $120 \mathrm{~h}(\mathrm{P}<0.05)$. Calculation of the number of TUNEL-reactive hepatocyte nuclei $24(37 \pm 2.5 / \mathrm{FOV}), 72(22 \pm 2.0 / \mathrm{FOV})$ and $120 \mathrm{~h}(10.5 \pm 1.8 / \mathrm{FOV})$ following intravenous transplantation of BMSCs indicated that the number of apoptotic hepatocyte nuclei was reduced compared with that of the control group.
The expression levels of apoptosis-associated proteins were detected by western blotting (Fig. 5A). As shown in Fig. 5B, in the control group, the protein expression of Bax was decreased with time, and was significantly decreased following transplantation of BMSCs. Furthermore, the Bcl-2 expression levels were significantly higher in the BMSC treatment group, compared with those of the control group (Fig. 5C). Differences between the two groups were significant at 24,72 and $120 \mathrm{~h}$ post-transplantation. These results indicated that BMSCs may exert protective effects against hepatocyte apoptosis following liver injury induced by LPS and D-Gal in a rat model.

BMSCs enhance liver regeneration. The induction of endogenous repair programs is a protective mechanism of BMSCs against acute liver injury. The number of PCNA-reactive hepatocytes in the BMSC treatment group and the control group was determined in order to evaluate the proliferative effect of BMSCs in the liver. The number of PCNA-positive hepatocytes increased in a time-dependent manner in the control and treatment groups (Fig. 6Aa-c). However, fewer PCNA-positive hepatocytes were expressed in the control group at 24, 72 and $120 \mathrm{~h}$, compared with that of the BMSC treatment group (Fig. 6A and B). As indicated in Fig. 6B, the number of PCNA-positive hepatocytes reached their peak $120 \mathrm{~h}$ following BMSC treatment. The number of proliferating hepatocytes was increased 1.71- and 1.97-fold in the BMSC 
A

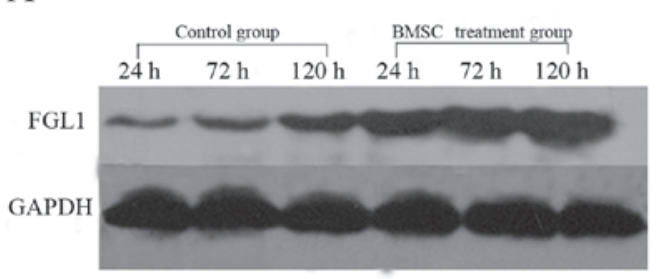

$\mathrm{B}$

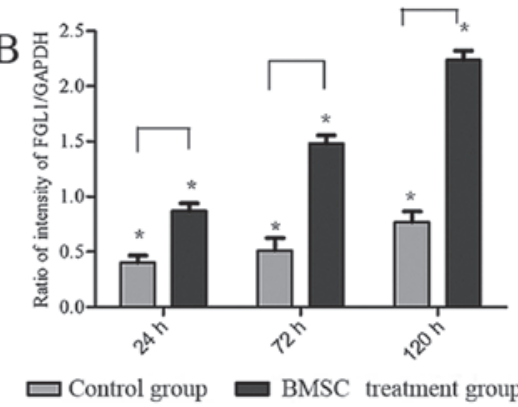

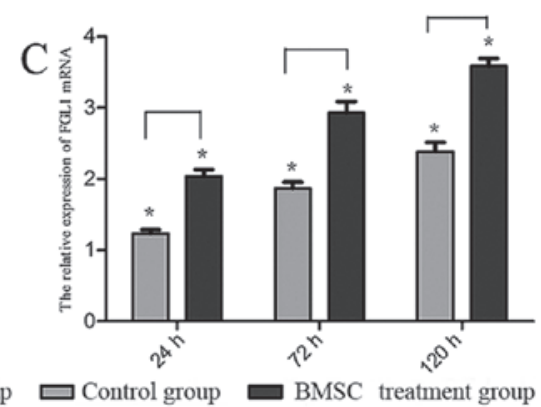

Figure 7. FGL1 protein and mRNA expression in the liver. (A) Protein expression levels of FGL1 were determined by western blotting. (B) Quantification of levels of FGL1 protein expression. (C) Relative FGL1 mRNA expression in the liver determined by reverse transcription-quantitative polymerase chain reaction. GAPDH was employed as an internal control. Values are expressed as the mean \pm standard deviation; ${ }^{*} \mathrm{P}<0.05$. FGL1, fibrinogen-like-protein 1; BMSCs bone marrow-derived mesenchymal stem cells; mRNA, messenger RNA.
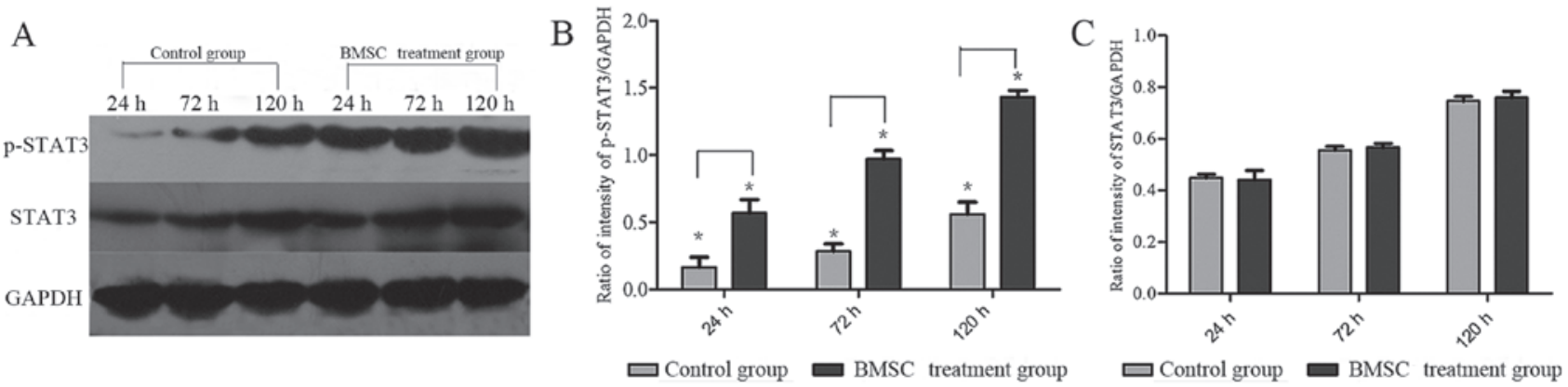

Figure 8. Levels of p-STAT3 and total STAT3 in liver tissues. (A) Protein expression levels of p-STAT3 and STAT3 were determined by western blotting (B) Quantification of levels of p-STAT3 protein expression. (C) Quantification of levels of STAT3 protein expression. GAPDH was employed as an internal control. Values are presented as the mean \pm standard deviation; " $\mathrm{P}<0.05$. STAT3, signal transducer and activator of transcription 3; p-, phosphorylated; BMSC, bone marrow derived stem cell; FOV, field of view.

treatment group, compared with that of the control group at 72 and 120 h post-treatment, respectively. Furthermore, these differences were demonstrated to be statistically significant. This result indicated that BMSCs were able to enhance liver regeneration following acute liver injury in a rat model.

BMSCs enhance FGL1 $\mathrm{mRNA}$ and protein expression in the liver. The expression levels of FGL1 protein (Fig. 7A and B) and mRNA (Fig. 7C) were detected by western blotting and qPCR analysis, respectively. The expression of FGL1 was elevated in the control group, indicating that FGL1 activity was induced at an early stage of liver damage and elevated by spontaneous regeneration; however, the BMSC treatment group exhibited significantly higher expression of FGL1 than that of the control group (Fig. 7B) at identical time-points (24, 72 and 120 h). As revealed in Fig. 7C, following treatment with BMSCs, the level of FGL1 mRNA was significantly augmented, compared with that of the control group at the same time-point. The degrees of growth were 1.75- $(\mathrm{P}<0.05)$, 2.1- $(\mathrm{P}<0.05)$ and 2.3-fold $(\mathrm{P}<0.05)$ greater at 24,72 and $120 \mathrm{~h}$ post-treatment, respectively.

BMSCs enhance p-STAT3 expression in liver tissue. To further elucidate the protective mechanisms of BMSC administration, the protein expression levels of STAT3 and p-STAT3 were evaluated by western blot analysis (Fig. 8A). The results revealed that the expression of p-STAT3 was significantly elevated in the BMSC treatment group, compared with that of the control group at the same time-point $(\mathrm{P}<0.05$; Fig. 8B); whereas no significant difference was detected in the expression of STAT3 between the two groups (Fig. 8C).

\section{Discussion}

The results of the present study revealed that intravenous BMSC transplantation exerted a protective effect against acute liver injury through increasing proliferation and decreasing hepatocyte apoptosis. The underlying mechanism may be in part due to the enhanced expression of FGL1 and activation of STAT3 signal transduction induced by BMSCs, which may alleviate the functional deterioration of the liver induced following acute injury.

Acute liver injury, induced by viruses (28), ethanol (29) and toxicity (30), is a growing health problem worldwide, and is associated with numerous complications throughout disease progression. Accumulating evidence suggests that liver injury is characterized by sequential, extensive activation of proteins and molecules $(31,32)$, and that maintenance of the apoptosis-regeneration balance has a vital role in modulating the process of liver recovery (33).

The potential therapeutic effects of BMSC transplantation have been verified by promising results observed in the treatment of acute liver injury $(34,35)$. Recently, studies have demonstrated that BMSCs reinforce liver regeneration and have significant suppressive effects on hepatocyte apoptosis (36-38). BMSCs suppress proapoptotic protein expression 
and enhance anti-apoptotic protein expression, consequently attenuating hepatocyte degeneration and subsequent apoptosis, as well as accelerating hepatocyte regeneration (39). Novel therapeutic approaches may be developed for the treatment of acute liver injury based on the results of the present study, which indicated that BMSCs suppressed the elevation of proapoptotic factor Bax, whilst upregulating the expression of anti-apoptotic factor Bcl-2, indicating that BMSCs were able to inhibit hepatocyte apoptosis. Furthermore, it was confirmed that BMSCs significantly enhanced the expression of FGL1, which possesses mitogenic activity in hepatocytes.

FGL1, a liver-specific protein found in humans and rats, was initially discovered in a rat model and is associated with the fibrinogen family (20). Research has indicated that FGL1 exerts multiple hepatic protective functions by promoting hepatocyte proliferation and decreasing the rate of apoptosis. A previous study indicated that administration of FGL1 to animals following D-Gal treatment significantly reversed liver injury by promoting hepatocyte proliferation and reducing liver apoptosis, while knockdown of endogenous FGL1 expression exacerbated liver injury $(22,23)$. Concurrent with these results, the present study indicated that the level of FGL1 was enhanced $72 \mathrm{~h}$ following the induction of liver injury, indicating that FGL1 was activated upon induction of spontaneous liver regeneration. Compared with that of the control group, the expression of FGL1 in the BMSC treatment group at the identical time-point $(24,72$ and $120 \mathrm{~h})$ was significantly higher, suggesting that BMSCs may have a role in the elevation of endogenous FGL1 expression. In addition, this elevation of FGL1 was accompanied by a decrease in the level of apoptosis and enhanced PCNA expression following liver injury, which revealed that FGL1 may attenuate the effects of liver injury by preventing hepatocyte apoptosis and enhancing liver regeneration. It was therefore postulated that the intravenous transplantation of BMSCs may exert protective effects by enhancing the expression of FGL1. Furthermore, it was previously reported that FGL1 was only able to influence normal liver cells (40), likely due to the existence of the relevant receptor in normal liver cells. Hence, intravenous transplantation of BMSCs may enhance the number and/or affinity of FGL1 receptors, although this hypothesis requires further investigation. Overall, it was demonstrated that BMSCs upregulated the expression of FGL1, which has a significant role in the elevation of liver regeneration and inhibition of hepatocyte apoptosis, and ultimately attenuated acute liver injury. However, further research is required in order to elucidate the exact mechanisms underlying these effects.

Previous studies have indicated that the STAT3 signal transduction pathway is involved in the liver injury regeneration and apoptosis mechanisms (40-42). Lou et al (43) revealed that hepatocyte-specific STAT3-deficient mice suffered more severe liver damage in a warm ischemia/reperfusion rat model. In order to evaluate the potential mechanisms by which BMSCs ameliorate liver injury, the protein expression levels of STAT3 and p-STAT3 were examined. STAT3 and p-STAT3 expression levels were increased in the BMSC treatment and control groups with time, demonstrating that STAT3 and p-STAT3 participated in LPS- and D-Gal-induced liver injury. However, the data revealed no significant differences in the expression levels of STAT3 between the two groups, suggesting that STAT3 may not be directly associated with the therapeutic effect of BMSCs. Notably, statistical differences in the expression levels of p-STAT3 were detected between the two groups, indicating that p-STAT3 had a role in the prevention of liver damage by BMSCs. p-STAT3 is the activated form of STAT3, a result of the phosphorylation of STAT3, which forms a dimer and is transferred to the nucleus in order to modulate the expression of anti-apoptotic gene Bax, Bcl-2 and cell cycle regulatory genes, including c-fos, c-myc and cyclin, and induce the progression of the cell cycle from $\mathrm{G}_{1}$ to $S$ phase, indicating its role in cell proliferation and apoptosis (44). Notably, the determination of the 5 ' flanking region of FGL1 in humans and rats indicated that there are DNA binding sites for STAT3 which are associated with the acceleration of promoter activity of FGL1 (45). It has been demonstrated that one of the pro-proliferative mechanisms of FLG1 is to maintain STAT3 phosphorylation; however, the specific underlying cross-talk remains to be elucidated and therefore further research is required.

In conclusion, the results of the present study demonstrated that BMSCs were therapeutically effective in the treatment of acute liver injury via positive regulation of the expression of endogenous FGL1 and activation of the STAT3 signaling pathway. These properties of BMSCs merit further clinical research for the development of novel therapeutic strategies for the treatment of acute liver injury.

\section{Acknowledgements}

The present study was supported by grants from the Scientific Research Foundation of Wenzhou, Zhejiang Province, China(grant nos. Y20100180 and Y20140073), the Medicine Health Project of Zhejiang Province, China (grant no. 2014KYB155) and Five-year Science and Technology Major Projects (grant no. 2012ZX10002004-010). The funders had no role in the study design, data collection and analysis, decision to publish or preparation of the manuscript.

\section{References}

1. Gitto S, Micco L, Conti F, Andreone P and Bernardi M: Alcohol and viral hepatitis: a mini-review. Dig Liver Dis 41: 67-70, 2009.

2. Leise MD, Poterucha JJ and Talwalkar JA: Drug-induced liver injury. Mayo Clin Proc 89: 95-106, 2014.

3. Bernal W and Wendon J: Acute liver failure. N Engl J Med 369: 2525-2534, 2013

4. Rai R: Liver transplantatation- an overview. Indian J Surg 75: 185-191, 2013.

5. Åberg F, Isoniemi $\mathrm{H}$ and Höckerstedt K: Long-term results of liver transplantation. Scand J Surg 100: 14-21, 2011.

6. Esrefoglu M: Role of stem cells in repair of liver injury: experimental and clinical benefit of transferred stem cells on liver failure. World J Gastroenterol 19: 6757-6773, 2013.

7. Wesson RN and Cameron AM: Stem cells in acute liver failure. Adv Surg 45: 117-130, 2011.

8. ReisLA,BorgesFT,Simões MJ,Borges AA, Sinigaglia-Coimbra R and Schor N: Bone marrow-derived mesenchymal stem cells repaired but did not prevent gentamicin-induced acute kidney injury through paracrine effects in rats. PloS One 7: e44092, 2012.

9. Cai B, Zhu S, Li J, Chen N, Liu Y and Lu Y: Bone marrow-derived mesenchymal stem cells protected rat cardiomyocytes from premature senescence. Int J Cardiol 154: 180-182, 2012.

10. van Haaften T, Byrne R, Bonnet S, et al: Airway delivery of mesenchymal stem cells prevents arrested alveolar growth in neonatal lung injury in rats. Am J Respir Crit Care Med 180: $1131-1142,2009$. 
11. Wang SP, Wang ZH, Peng DY, Li SM, Wang $\mathrm{H}$ and Wang $\mathrm{XH}$ : Therapeutic effect of mesenchymal stem cells in rats with intracerebral hemorrhage: Reduced apoptosis and enhanced neuroprotection. Mol Med Rep 6: 848-854, 2012.

12. Han Y, Lan N, Pang C and Tong X: Bone marrow-derived mesenchymal stem cells enhance cryopreserved trachea allograft epithelium regeneration and vascular endothelial growth factor expression. Transplantation 92: 620-626, 2011.

13. Gabrielyan A, Knaak S, Gelinsky M, Arnhold S and Rösen-Wolff A: Hypoxia-conditioned media allows species-specific attraction of bone marrow stromal cells without need for recombinant proteins. BMC Vet Res 10: 56, 2014.

14. Seyfried DM, Han Y, Yang D, et al: Localization of bone marrow stromal cells to the injury site after intracerebral hemorrhage in rats. J Neurosurg 112: 329-335, 2010.

15. $\mathrm{Xu}$ YQ and Liu ZC: Therapeutic potential of adult bone marrow stem cells in liver disease and delivery approaches. Stem Cell Rev 4: 101-112, 2008.

16. Mezey E: The therapeutic potential of bone marrow-derived stromal cells. J Cell Biochem 112: 2683-2687, 2011.

17. Meirelles Lda S,Fontes AM,Covas DT and Caplan AI:Mechanisms involved in the therapeutic properties of mesenchymal stem cells. Cytokine Growth Factor Rev 20: 419-427, 2009.

18. Uccelli A, Moretta L and Pistoia V: Mesenchymal stem cells in health and disease. Nat Rev Immunol 8: 726-736, 2008.

19. Xagorari A, Siotou E, Yiangou M, et al: Protective effect of mesenchymal stem cell-conditioned medium on hepatic cell apoptosis after acute liver injury. Int J Clin Exp Pathol 6 : 831-840, 2013

20. Yamamoto T, Gotoh M, Sasaki H, Terada M, Kitajima M and Hirohashi S: Molecular cloning and initial characterization of a novel fibrinogen-related gene, HFREP-1. Biochem Biophys Res Commun 193: 681-687, 1993.

21. Cao MM, Xu WX, Li CY, et al: Hepassocin regulates cell proliferation of the human hepatic cells L02 and hepatocarcinoma cells through different mechanisms. J Cell Biochem 112: 2882-2890, 2011.

22. Li CY, Cao CZ, Xu WX, et al: Recombinant human hepassocin stimulates proliferation of hepatocytes in vivo and improves survival in rats with fulminant hepatic failure. Gut 59: 817-826, 2010.

23. Yan J, Ying H, Gu F, et al: Cloning and characterization of a mouse liver-specific gene mfrep-1, up-regulated in liver regeneration. Cell Res 12: 353-361, 2002.

24. Chang YP, Hong HP, Lee YH and Liu IH: The canine epiphyseal-derived mesenchymal stem cells are comparable to bone marrow derived-mesenchymal stem cells. J Vet Med Sci: Nov 12, 2014 (Epub ahead of print).

25. Zhu X, Tan T, Yao W, et al: Optimization of the method for isolating and culturing rat mesenchymal stem cells. Nan Fang Yi Ke Da Xue Xue Bao 34: 1621-1626, 2014 (In Chinese).

26. Derveaux S, Vandesompele J and Hellemans J: How to do successful gene expression analysis using real-time PCR Methods 50: 227 230, 2010.

27. Rujiter JM, Pfaffl MW, Zhao S, et al: Evaluation of qPCR curve analysis methods for reliable biomarker discovery: Bias, resolution, precision, and implications. Methods 59: 32-46, 2013.
28. Huang SN, Chen TC, Tsai SL and Liaw YF: Histopathology and pathobiology of hepatotropic virus-induced liver injury. J Gastroenterol Hepatol 12: S195-S217, 1997.

29. Teplova VV, Belosludtsev KN, Belosludtseva NV and Kholmukhamedov EL: Mitochondria and hepatotoxicity of ethanol. Biofizika 55: 1038-1047, 2010 (In Russian).

30. Lopez AM and Hendrickson RG: Toxin-induced hepatic injury. Emerg Med Clin North Am 32: 103-125, 2014.

31. Kaplowitz N: Biochemical and cellular mechanisms of toxic liver injury. Semin Liver Dis 22: 137-144, 2002.

32. Muntané J,GonzálezR,RanchalI,ColladoJA,López-SánchezLM, Herencia C, Rodríguez-Ariza A, Rafael Muñoz-Castañeda JR and de la Mata M: Mechanisms of liver cell injury. Rev Esp Enferm Dig 99: 405-410, 2007 (In Spanish).

33. Galun E and Axelrod JH: The role of cytokines in liver failure and regeneration: potential new molecular therapies. Biochim Biophys Acta 1592: 345-358, 2002.

34. Chen XW, Zhu DJ, Ju YL and Zhou SF: Therapeutic effect of transplanting magnetically labeled bone marrow stromal stem cells in a liver injury rat model with $70 \%$-hepatectomy. Med Sci Monit 18: BR375-BR382, 2012.

35. Sun K, Xie X, Xie J, et al: Cell-based therapy for acute and chronic liver failures: Distinct diseases, different choices. Sci Rep 4: 6494, 2014

36. Hang HL and Xia Q: Role of BMSCs in liver regeneration and metastasis after hepatectomy. World J Gastroenterol 20: 126-132, 2014.

37. Li J, Zhang L, Xin J, et al: Immediate intraportal transplantation of human bone marrow mesenchymal stem cells prevents death from fulminant hepatic failure in pigs. Hepatology 56: 1044-1052, 2012

38. Yuan S, Jiang T, Sun L, Zheng R, Ahat N and Zhang Y: The role of bone marrow mesenchymal stem cells in the treatment of acute liver failure. Biomed Res Int 2013: 251846, 2013.

39. Zhu X, He B, Zhou X and Ren J: Effects of transplanted bone-marrow-derived mesenchymal stem cells in animal models of acute hepatitis. Cell Tissue Res 351: 477-486, 2013.

40. Ozaki M: Role of jak/STAT3 and PI3-K/Akt pathways in liver injury and regeneration. Seikagaku 80: 399-408, 2008 (In Japanese).

41. Li M, Zhou X, Mei J, et al: Study on the activity of the signaling pathways regulating hepatocytes from G0 phase into G1 phase during rat liver regeneration. Cell Mol Biol Lett 19: 181-200, 2014.

42. da Silva CG, Studer P, Skroch M, et al: A20 promotes liver regeneration by decreasing SOCS3 expression to enhance IL-6/STAT3 proliferative signals. Hepatology 57: 2014-2025, 2013.

43. Lou LX, Uemura T, Mani H, et al: Endogenous signal transducer and activator of transcription 3 is required for the protection of hepatocytes against warm ischemia/reperfusion injury. Liver Transpl 19: 1078-1087, 2013.

44. Li W, Liang X, Kellendonk C, Poli V and Taub R: STAT3 contributes to the mitogenic response of hepatocytes during liver regeneration. J Biol Chem 277: 28411-28417, 2002.

45. Liu Z and Ukomadu C: Fibrinogen-like protein 1, a hepatocyte derived protein is an acute phase reactant. Biochem Biophys Res Commun 365: 729-734, 2008. 